\title{
Cross section systematics for the lightest Bi and Po nuclei produced in complete fusion reactions with heavy ions
}

\author{
A. N. Andreyev, ${ }^{1,2,3}$ D. Ackermann, ${ }^{4,5}$ S. Antalic, ${ }^{6}$ I. G. Darby, ${ }^{2}$ S. Franchoo,${ }^{5,7}$ F. P. Heßberger, ${ }^{4}$ S. Hofmann, ${ }^{4,8}$ \\ M. Huyse, ${ }^{3}$ P. Kuusiniemi, ${ }^{4}$ B. Lommel, ${ }^{4}$ B. Kindler, ${ }^{4}$ R. Mann, ${ }^{4}$ G. Münzenberg, ${ }^{4,5}$ R. D. Page, ${ }^{2}$ S. Saro, ${ }^{6}$ B. Sulignano, ${ }^{4}$ \\ B. Streicher, ${ }^{6}$ K. Van de Vel, ${ }^{3, *}$ P. Van Duppen, ${ }^{3}$ and D. R. Wiseman ${ }^{2}$ \\ ${ }^{1}$ TRIUMF, 4004 Wesbrook Mall, Vancouver, BC, V6T 2A3, Canada \\ ${ }^{2}$ Department of Physics, Oliver Lodge Laboratory, University of Liverpool, Liverpool L69 7ZE, UK \\ ${ }^{3}$ Instituut voor Kern- en Stralingsfysica, University of Leuven, Celestijnenlaan 200 D, B-3001 Leuven, Belgium \\ ${ }^{4}$ Gesellschaft fur Schwerionenforschung (GSI), Planckstrasse 1, D-64291 Darmstadt, Germany \\ ${ }^{5}$ Institut für Physik, Johannes Gutenberg-Universität, Staudingerweg 7, D-55099 Mainz, Germany \\ ${ }^{6}$ Department of Nuclear Physics, Comenius University, Bratislava, Slovakia \\ ${ }^{7}$ ISOLDE, CERN, CH-1211 Geneve 23, Switzerland \\ ${ }^{8}$ Physikalisches Institut, J.W. Goethe-Universität, D-60054 Frankfurt, Germany
}

(Received 8 April 2005; published 29 July 2005)

\begin{abstract}
The production of the very neutron-deficient nuclides ${ }^{184-192} \mathrm{Bi}$ and ${ }^{186-192} \mathrm{Po}$ in the vicinity of the neutron midshell at $N=104$ has been studied by using heavy-ion-induced complete fusion reactions in a series of experiments at the velocity filter SHIP. The cross sections for the $x n$ and $p x n$ evaporation channels of the ${ }^{46} \mathrm{Ti}+{ }^{144} \mathrm{Sm} \rightarrow{ }^{190} \mathrm{Po}^{*},{ }^{98} \mathrm{Mo}+{ }^{92} \mathrm{Mo} \rightarrow{ }^{190} \mathrm{Po}^{*},{ }^{50,52} \mathrm{Cr}+{ }^{142} \mathrm{Nd} \rightarrow{ }^{192,194} \mathrm{Po}^{*}$, and ${ }^{94,95} \mathrm{Mo}+{ }^{93} \mathrm{Nb} \rightarrow{ }^{187,188} \mathrm{Bi}^{*}$ reactions were measured. The results obtained, together with the previously known cross section data for the heavier Bi and Po nuclides, are compared with the results of statistical model calculations carried out with the HIVAP code. It is shown that a satisfactory description of the experimental data requires a significant (up to 35\%) reduction of the theoretical fission barriers. The optimal reactions for production of the lightest $\mathrm{Bi}$ and Po isotopes are discussed.
\end{abstract}

DOI: 10.1103/PhysRevC.72.014612

PACS number(s): 24.10.Pa, 23.60.+e, 27.70.+q, 27.80.+w

\section{INTRODUCTION}

Complete fusion reactions between heavy nuclei are a suitable tool for the investigation of both the thermodynamic characteristics of nuclear matter (e.g., the temperature and decay widths of hot nuclei) and its dynamical characteristics (e.g., the rate of energy dissipation, nuclear viscosity, etc.). Often in such studies the experimental data, such as pre/post-scission particle multiplicities, excitation functions for evaporation residue (ER), and/or fission channels, are compared with the results of different theoretical approaches with the aim of deriving the values of the parameters introduced within the model and to see how these parameters depend on experimentally controlled conditions such as the nuclear excitation energy, angular momentum transfer, and entrance channel asymmetry. A particular example relevant to our study is the rotating liquid drop model (RLDM) [1] and its modifications (see [2] and references therein), which are widely used to describe the excited nuclei produced in complete fusion reactions. As part of the statistical model of compound nucleus $(\mathrm{CN})$ formation and subsequent decay via the competition between particle evaporation, $\gamma$-ray emission, and fission, the RLDM is often used to fit the experimental ER and/or fission cross sections by exploiting only two parameters: $C_{f}$, a scaling factor for the RLDM fission barrier, and $\tilde{a}_{f} / \tilde{a}_{v}$, the ratio of

${ }^{*}$ Present address: Flemish Institute for Technological Research (VITO), Boeretang 200, B-2400 Mol, Belgium. the asymptotic level density parameters at the saddle point to that at the equilibrium deformation of the ER. Over the last two decades, this approach has been extensively and successfully used by many authors; a thorough discussion of its advantages and drawbacks can be found, for example, in Refs. [3-5] for $A=140-180$ nuclei and in Refs. [6-13] for nuclei in the Hg-U region. Some of the studies also included in the analysis the evaporation particle multiplicities [6,14], which proved to be useful for improving the parameters in the calculations. It is also worth noting that recently a new method to extract the fission barriers of neutron-deficient nuclei in the Ra-U region was demonstrated that uses the electromagnetic-induced fission of secondary radioactive ion beams produced in high-energy fragmentation reactions [15].

The neutron-deficient $\mathrm{Bi}$ and $\mathrm{Po}$ nuclei $(Z=83,84)$ represent an interesting case for such studies, having a high fissility with typical values of $Z^{2} / A \sim 35-38$ for $A=$ 185-200, which are comparable to the value of $Z^{2} / A \sim 36$ for ${ }^{235} \mathrm{U}$. Consequently, their production cross sections are especially sensitive to the choice of parameters in the model calculations, and although the cross sections become quite low, they are still measurable with the necessary precision. Another attractive feature of these nuclei is that most of them can be produced in a variety of complete fusion reactions, from very asymmetric to completely symmetric reactions in the entrance channel. Examples are the earlier studies of the ${ }^{164} \mathrm{Dy}\left({ }^{40} \mathrm{Ar}, x n\right){ }^{204-x} \mathrm{Po},{ }^{160} \mathrm{Dy}\left({ }^{40} \mathrm{Ar}, x n\right){ }^{200-x} \mathrm{Po}[16,17]$ and ${ }^{116} \mathrm{Cd}\left({ }^{84} \mathrm{Kr}, x n\right){ }^{200-x}$ Po reactions [17]. The analysis of cross sections for the $x n$ deexcitation channel of the two latter 
TABLE I. Reactions used in this work to produce neutron-deficient $\mathrm{Pb}, \mathrm{Bi}$, and Po nuclides. Also given are the beam energy range $E_{\text {beam }}$, typical beam intensity $I_{\text {beam }}$, and target thickness.

\begin{tabular}{lcccccc}
\hline \hline $\begin{array}{l}\text { Compound } \\
\text { nucleus }\end{array}$ & Reaction & Studied nuclei & $\begin{array}{c}E_{\text {beam }} \\
(\mathrm{MeV})\end{array}$ & $\begin{array}{c}I_{\text {beam }} \\
(\mathrm{pnA})\end{array}$ & $\begin{array}{c}\text { Target thickness } \\
\left(\mu \mathrm{g} / \mathrm{cm}^{2}\right)\end{array}$ \\
\hline${ }^{194} \mathrm{Po}^{*}$ & ${ }^{52} \mathrm{Cr}+{ }^{142} \mathrm{Nd}^{\mathrm{a}}$ & ${ }^{188-192} \mathrm{Po},{ }^{187-192} \mathrm{Bi}$ & $220-310$ & $\sim 300$ & $290(20)$ \\
${ }^{192} \mathrm{Po}^{*}$ & ${ }^{50} \mathrm{Cr}+{ }^{142} \mathrm{Nd}^{\mathrm{a}}$ & ${ }^{188,189} \mathrm{Po},{ }^{186-190} \mathrm{Bi}$ & $230-285$ & $\sim 50$ & $290(20)$ & {$[20,24,25,30]$} \\
${ }^{190} \mathrm{Po}^{*}$ & ${ }^{46} \mathrm{Ti}+{ }^{144} \mathrm{Sm}^{\mathrm{b}}$ & $186,187 \mathrm{Po},{ }^{186,187} \mathrm{Bi}$ & $202-242$ & $\sim 150$ & $300-400$ & {$[25,26]$} \\
${ }^{190} \mathrm{Po}^{*}$ & ${ }^{98} \mathrm{Mo}+{ }^{92} \mathrm{Mo}^{\mathrm{c}}$ & ${ }^{186-188} \mathrm{Bi}$ & $427-460$ & $\sim 10$ & $500-700$ & This work \\
${ }^{188} \mathrm{Bi}^{*}$ & ${ }^{95} \mathrm{Mo}+{ }^{93} \mathrm{Nb}^{\mathrm{d}}$ & ${ }^{185-187} \mathrm{Bi},{ }^{183-187} \mathrm{~Pb}$ & $375-456$ & $\sim 10$ & $900(50)$ & {$[23,31]$} \\
${ }^{187} \mathrm{Bi}^{*}$ & ${ }^{94} \mathrm{Mo}+{ }^{93} \mathrm{Nb}^{\mathrm{d}}$ & ${ }^{184,185} \mathrm{Bi},{ }^{183-185} \mathrm{~Pb}$ & $405-450$ & $\sim 10$ & $900(50)$ & {$[23]$} \\
\hline \hline
\end{tabular}

${ }^{\text {a The }}{ }^{142} \mathrm{NdF}_{3}$ material (isotopic enrichment $99.8 \%$ ) was evaporated on the $50 \mu \mathrm{g} / \mathrm{cm}^{2}$ carbon backing and covered with a $10 \mu \mathrm{g} / \mathrm{cm}^{2}$ layer of carbon to improve the cooling of the target and to reduce the sputtering of the target material.

${ }^{\mathrm{b}} \mathrm{The}^{144} \mathrm{SmF}_{3}$ material (isotopic enrichment 96.47\%) was evaporated on the $40 \mu \mathrm{g} / \mathrm{cm}^{2}$ carbon backing and covered with a $10 \mu \mathrm{g} / \mathrm{cm}^{2}$ layer of carbon.

${ }^{\mathrm{c}}$ Self-supporting target, $97.37 \%{ }^{92}$ Mo enrichment, rolled metallic.

${ }^{\mathrm{d}}$ Self-supporting target, $100 \%{ }^{93} \mathrm{Nb}$ natural abundance, rolled metallic.

reactions leading to the same $\mathrm{CN}^{200} \mathrm{Po}^{*}$ [17] has shown that for excitation energies above the respective Coulomb barriers, the shapes of the excitation functions and their positions on the excitation-energy axis practically coincide. Furthermore, it was demonstrated that the ratio of the cross sections for the channels with a given number of emitted neutrons is close to the ratio of the squares of the reduced de Broglie wavelengths in the entrance channels of these reactions, $\frac{\sigma_{x n}(\mathrm{Ar})}{\sigma_{x n}(\mathrm{Kr})} \approx \frac{\lambda^{2}(\mathrm{Ar})}{\lambda^{2}(\mathrm{Kr})}$. This supports the idea that there are no major differences in the formation and deexcitation of ${ }^{200} \mathrm{Po}^{*}$ formed by ${ }^{40} \mathrm{Ar}$ or by ${ }^{84} \mathrm{Kr}$.

These studies were further extended by measuring the $x n$ (and, in some cases, pxn) evaporation channels for the asymmetric reaction ${ }^{31} \mathrm{P}+{ }^{169} \mathrm{Tm} \rightarrow{ }^{200} \mathrm{Po}^{*}[10]$ at the electrostatic separator VASSILISSA (FLNR, Dubna) [12] and for the symmetric reaction ${ }^{100} \mathrm{Mo}+{ }^{100} \mathrm{Mo} \rightarrow{ }^{200} \mathrm{Po}^{*}$ [9] at the velocity filter SHIP (GSI, Darmstadt) [18,19]. A comparative study [10] of the four above-mentioned reactions resulting in the same compound nucleus ${ }^{200} \mathrm{Po}^{*}$ led to the important conclusion that above the respective Coulomb barriers, no fusion hindrance was observed, and both the magnitude and the excitation-energy dependence of the $x n$ evaporation channel of the ${ }^{200} \mathrm{Po}^{*}$ compound nucleus do not depend on the mass of the projectiles up to complete symmetry in the entrance channel. Furthermore, it was concluded that a significant (up to $25-30 \%$ ) reduction of the liquid drop (LD) part of the fission barrier was necessary for a satisfactory description of the polonium cross sections within the framework of the statistical model. A similar conclusion was also derived from studies of the ${ }^{187-195} \mathrm{Bi}$ nuclei, produced in the $x n$ evaporation channels of different complete fusion reactions leading to bismuth compound nuclei [11]. More generally, the conclusion on the necessity to reduce the LD fission barriers in the statistical model calculations for the neutron-deficient nuclei agreed with the results of other works in the $A \sim 150$ and $\mathrm{Pb}-\mathrm{U}$ regions, see $[3,6,9-13,15]$ and references therein.

As an additional motivation, very neutron-deficient $\mathrm{Pb}$ Po nuclei are of interest due to the rich variety of nuclear spectroscopic phenomena they exhibit. For example, the double and triple shape coexistence phenomena were predicted and indeed recently observed at low excitation energy in some of these nuclei in vicinity of the neutron midshell at $N=104$ (see [20-22] and references therein). Therefore, for future progress with these nuclei having very low production cross sections (e.g., $\sim 0.2 \mathrm{nb}$ for ${ }^{186} \mathrm{Po}$, see below) it becomes crucial to have reliable cross section data or calculations when planning experiments.

Thus, the goal of the present investigation was to continue earlier cross section studies to even more neutron-deficient $\mathrm{Bi}$ and Po nuclides. By using the high sensitivity of the velocity filter SHIP, combined with high-intensity heavy-ion beams and specially designed large-area rotating targets, we were able to extend the cross section systematics to the new isotopes ${ }^{184} \mathrm{Bi}$ [23] and ${ }^{186-189} \mathrm{Po}$ [24-27]. Furthermore, in contrast to most of the previous works, both the $x n$ and $p x n$ evaporation channels of the studied reactions could be simultaneously investigated, which is important for the subsequent analysis within the statistical model.

\section{EXPERIMENTAL DETAILS}

\section{A. Experimental setup}

The list of reactions used in our studies is given in Table I. While the specific details on each experiment can be found in the corresponding references in Table I, for consistency of the discussion we provide here a short description of the method.

In all experiments, the pulsed beam ( $5 \mathrm{~ms}$ on/ $15 \mathrm{~ms}$ off $)$ was provided by the UNILAC heavy-ion accelerator of GSI. Eight targets made of a specific isotope (see Table I) were mounted on a target wheel, rotating synchronously with the UNILAC macropulsing. A few beam energies with a typical step of 7-10 MeV were used to allow excitation function measurements. The absolute beam energy was measured with an accuracy of $\pm 0.5 \%$.

After separation by the velocity filter SHIP [18], the ERs were implanted into a $300 \mu \mathrm{m}$ thick, $35 \times 80 \mathrm{~mm}^{2}$ 16-strip position-sensitive silicon detector (PSSD), where 
TABLE II. Branching ratios $b_{\alpha}$ for $\mathrm{Bi}$ and $\mathrm{Pb}$ nuclides used in this work. For nuclei with two $\alpha$-decaying isomeric states, the branching ratios for both are given, see references for details. For ${ }^{185} \mathrm{Bi}$, the sum of the proton and $\alpha$-branching ratios $b_{p}+b_{\alpha}$ is given.

\begin{tabular}{lcccr}
\hline \hline $\mathrm{Pb}$ isotope & $b_{\alpha}(\%)$ & Refs. & Bi isotope & $b_{\alpha}(\%)$ \\
\hline${ }^{187 m, g} \mathrm{~Pb}$ & $12(2), 9.5(2.0)$ & {$[32]$} & ${ }^{192 m, g} \mathrm{Bi}$ & $10(3), 12(5)$ \\
${ }^{186} \mathrm{~Pb}$ & $38(9)$ & {$[34]$} & ${ }^{191{ }^{g} \mathrm{Bi}}$ & {$[33]$} \\
${ }^{185 m, g} \mathrm{~Pb}$ & $34(25), 34(25)$ & {$[26]$} & ${ }^{190 m, g} \mathrm{Bi}$ & $51(10)$ \\
${ }^{184} \mathrm{~Pb}$ & $80(15)$ & {$[25,31]$} & ${ }^{185} \mathrm{Bi}$ & $70(9), 90_{-30}^{+10}$ \\
\hline \hline
\end{tabular}

their subsequent particle ( $\alpha$ and proton) decays were measured [28].

Three thin time-of-flight detectors [29] were installed in front of the PSSD system allowing us to distinguish the reaction products from the scattered beam particles. More importantly, decay events in the PSSD could be distinguished from the implantation events by requiring an anticoincidence condition between the signal from the PSSD and the signal from at least one of these detectors.

\section{B. Determination of absolute cross sections}

Identification of most of the nuclei could be performed using their unique $\alpha$-decay energy and half-life. In some cases, e.g., for the new nuclides ${ }^{184} \mathrm{Bi}$ [23] and ${ }^{186-189} \mathrm{Po}$ $[24,27]$ produced with relatively low statistics, the method of genetically correlated events was used [28]. Examples of measured $\alpha$-decay spectra for each studied reaction can be found in the references in Table I.

The primary production rates for each nuclide of interest, and hence for each evaporation channel, were obtained from such spectra, then normalized to the beam dose and further corrected for branching ratios (where necessary) and the reaction-dependent SHIP transmission efficiency in order to deduce the cross sections.

The beam current was measured at a beam stop (a cooled copper plate) inside the SHIP; the stability of the target was controlled by two monitor silicon detectors which measured projectiles elastically scattered from the target.

Based on well-established systematics, the $\alpha$-branching ratio of $b_{\alpha}=100 \%$ was assumed for the nuclides ${ }^{186-192} \mathrm{Po}$, ${ }^{184,186-189} \mathrm{Bi}$, and ${ }^{183} \mathrm{~Pb}$, while the values for other nuclides studied in this work are given in Table II. For ${ }^{185} \mathrm{~Pb}$, the branching ratio of the low-spin isomer was recently estimated as $b_{\alpha}=34(25) \%$ in Ref. [26]; we assumed the same branching ratio for the high-spin isomer based on its similar transition properties.

The SHIP transmission efficiency was calculated using a Monte Carlo simulation (see details in Ref. [5]), and the resulting values of $40 \%$ and $60 \%$ were used for the Ti-, Cr-, and Mo-induced reactions, respectively. The same efficiency was assumed for the $x n$ and $p x n$ evaporation channels, while the efficiency for the $\alpha x n$ channel was a factor of 5-10 lower because of a broader ER angular distribution after the $\alpha$-particle emission. More details on the extraction of the absolute cross sections can be found in Ref. [7].

The measured excitation functions are shown in Figs. 1-3 for Po compound nuclei and later in Figs. 6-7 for Bi compound nuclei. Table III summarizes the measured maximum cross section values and optimal reactions which could be exploited to produce the ${ }^{186-192}$ Po isotopes by using stable beams and targets. These data will be discussed in detail in the relevant sections of the present paper. The typical overall (statistical and systematic) absolute uncertainty of most values in the vicinity of the excitation function maxima, considered preferentially in the statistical model calculations in Sec. III, is $\pm 40 \%$ and is not shown in the figures for the sake of their clarity. It is mostly determined by the combination of the systematic errors related to the target thickness and SHIP transmission efficiency, and by the uncertainties of the $\alpha$-decay branching ratios (for the nuclei in Table II). The statistical errors in the maxima of the excitation functions were usually less than 5\%. However, for the most neutron-deficient nuclides $\left({ }^{184,185} \mathrm{Bi}\right.$ and $\left.{ }^{186-188} \mathrm{Po}\right)$ and at the tails of the excitation functions, the statistical uncertainty could reach up to $20 \%$. The relative uncertainty of the cross sections is about $15 \%$. Cross sections for the $\alpha x n$ channels of the studied reactions could not be reliably deduced in most cases, mostly because of the larger uncertainty of the SHIP transmission efficiency for these channels and

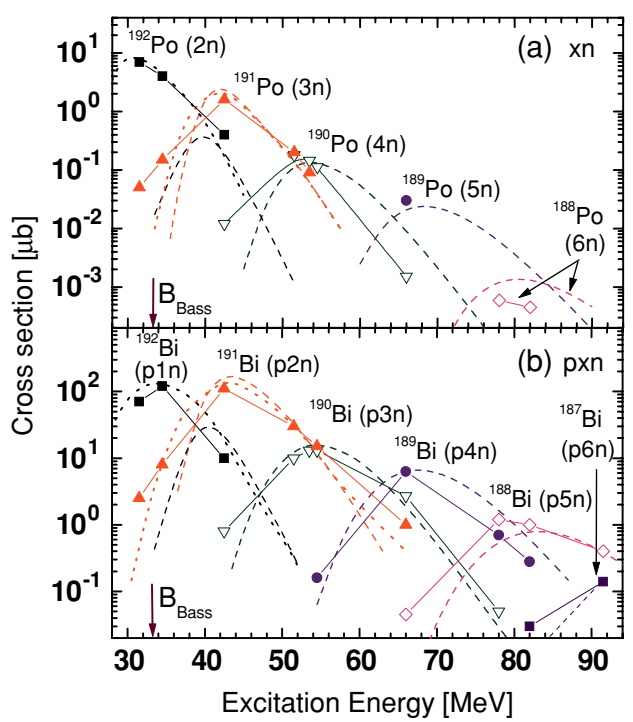

FIG. 1. (Color online) Absolute cross sections for the ${ }^{52} \mathrm{Cr}+{ }^{142} \mathrm{Nd} \rightarrow{ }^{194} \mathrm{Po}^{*}$ reaction: (a) $x n$ channels, (b) pxn channels. Experimental data are shown by the symbols connected with the solid lines. Calculated values $\left(C_{f}=0.68\right)$ are shown by dashed lines for the inverted parabola (IP) approximation for the barrier and by dotted lines for the barrier fluctuation (BF) method, see text for details. Bass barrier is indicated by vertical arrow. 


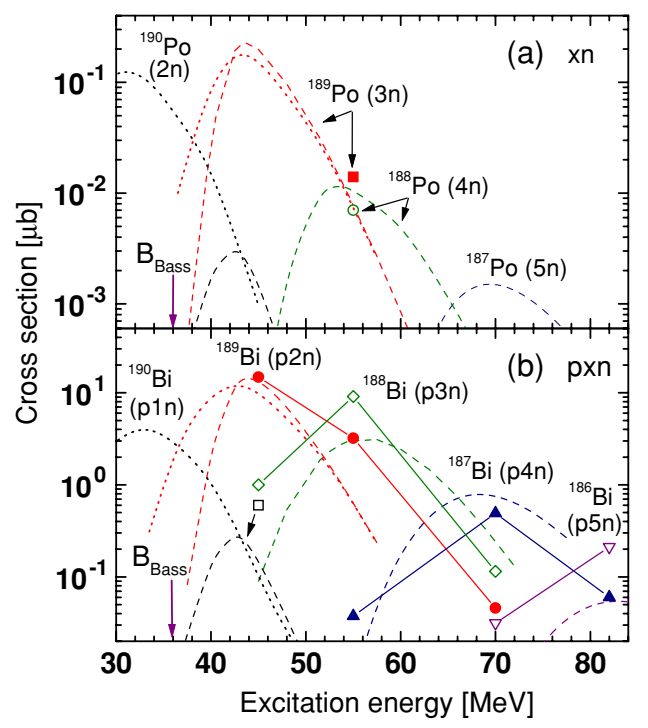

FIG. 2. (Color online) Same as Fig. 1, but for ${ }^{50} \mathrm{Cr}+{ }^{142} \mathrm{Nd} \rightarrow$ ${ }^{192} \mathrm{Po}^{*}$. Experimental data are shown by symbols; calculated values $\left(C_{f}=0.67\right)$, by dashed (IP variant) and dotted (BF variant) lines.

in the case of the Bi compound nuclei, the large uncertainties in the weak $\alpha$-decay branching ratios of the daughter $\mathrm{Tl}$ isotopes.

\section{HIVAP CALCULATIONS AND DISCUSSION OF THE DATA}

\section{A. Details of the calculations}

A few practical implementations of the statistical model exist, such as the codes HIVAP [8], JULIAN/PACE [4,39], ALICE [40], and their recent modifications. One would expect that comparable results should be obtained when an identical set of input parameters is used with different codes. However, in

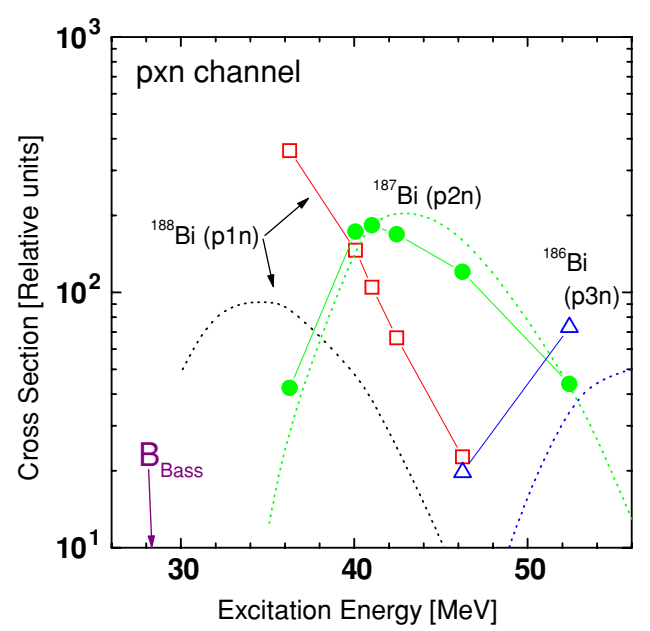

FIG. 3. (Color online) Relative production cross sections for the pxn channel of the ${ }^{98} \mathrm{Mo}+{ }^{92} \mathrm{Mo} \rightarrow{ }^{190} \mathrm{Po}^{*}$ reaction. Calculated values (BF variant) were normalized to the measured data at the maximum of ${ }^{187} \mathrm{Bi}$. See text for details.
TABLE III. Experimental and calculated cross sections for ${ }^{186-192}$ Po nuclei. Calculated values are denoted by HIVAP. Quoted errors of calculated values correspond to the 0.01 uncertainty of the $C_{f}$ values used, see discussion in text for details.

\begin{tabular}{lcccc}
\hline \hline Isotope & Reaction & Channel & $\begin{array}{c}\text { Cross } \\
\text { section } \\
(\mu b)\end{array}$ & Refs. \\
\hline${ }^{192} \mathrm{Po}$ & ${ }^{52} \mathrm{Cr}+{ }^{142} \mathrm{Nd}$ & $2 n$ & $8(2)$ & Fig. 1 and [25] \\
& ${ }^{36} \mathrm{Ar}+{ }^{160} \mathrm{Dy}$ & $4 n$ & $\sim 10$ & {$[36]$} \\
& ${ }^{49} \mathrm{Ti}+{ }^{147} \mathrm{Sm}$ & $4 n$ & $7.4(2.4)$ & HIVAP \\
& ${ }^{51} \mathrm{~V}+{ }^{144} \mathrm{Sm}$ & $p 2 n$ & $0.8(2)$ & [37] \\
${ }^{191} \mathrm{Po}$ & ${ }^{52} \mathrm{Cr}+{ }^{142} \mathrm{Nd}$ & $3 n$ & $1.6(4)$ & Fig. 1 and [25] \\
& ${ }^{52} \mathrm{Cr}+{ }^{142} \mathrm{Nd}$ & $3 n$ & $2.5(8)$ & HIVAP, Fig. 1 \\
& ${ }^{50} \mathrm{Ti}+{ }^{144} \mathrm{Sm}$ & $3 n$ & $2.9(9)$ & HIVAP \\
${ }^{190} \mathrm{Po}$ & ${ }^{52} \mathrm{Cr}+{ }^{142} \mathrm{Nd}$ & $4 n$ & $0.15(4)$ & Fig. 1 and [25] \\
& ${ }^{49} \mathrm{Ti}+{ }^{144} \mathrm{Sm}$ & $3 n$ & $0.25(8)$ & [38] \\
& ${ }^{49} \mathrm{Ti}+{ }^{144} \mathrm{Sm}$ & $3 n$ & $0.33(11)$ & HIVAP \\
${ }^{189} \mathrm{Po}$ & ${ }^{52} \mathrm{Cr}+{ }^{142} \mathrm{Nd}$ & $5 n$ & $0.03(1)$ & Fig. 1 and \\
& & & & [25,26] \\
& ${ }^{50} \mathrm{Cr}+{ }^{142} \mathrm{Nd}$ & $3 n$ & $0.23(8)$ & HIVAP, Fig. 2 \\
& ${ }^{48} \mathrm{Ti}+{ }^{144} \mathrm{Sm}$ & $3 n$ & $0.24(8)$ & HIVAP \\
${ }^{188} \mathrm{Po}$ & ${ }^{52} \mathrm{Cr}+{ }^{142} \mathrm{Nd}$ & $6 n$ & $0.0006(3)$ & Fig. 1 \\
& ${ }^{50} \mathrm{Cr}+{ }^{142} \mathrm{Nd}$ & $4 n$ & $0.008(4)$ & Fig. 2 \\
& ${ }^{47} \mathrm{Ti}+{ }^{144} \mathrm{Sm}$ & $3 n$ & $0.03(1)$ & HIVAP \\
${ }^{187} \mathrm{Po}$ & ${ }^{46} \mathrm{Ti}+{ }^{144} \mathrm{Sm}$ & $3 n$ & $0.0010(3)$ & {$[27]$} \\
${ }^{186} \mathrm{Po}$ & ${ }^{46} \mathrm{Ti}+{ }^{144} \mathrm{Sm}$ & $4 n$ & $0.00020(5)$ & [27] \\
\hline \hline
\end{tabular}

practice the codes use different parametrizations, e.g., particle transmission coefficients, level density expressions, fission barriers, and other parameters, some of which can only be estimated or extracted from other (semi-)phenomenological approaches. Therefore, care should be taken when using and comparing the results of different calculations, especially when dealing with the nuclei with low proton binding energy and/or low fission barriers.

In this work, the statistical model code HIVAP [8] was used because its parameters were carefully adjusted by the authors of the code to a large body of experimental data covering a broad region of nuclei, in particular including heavy and strongly fissile nuclei. With the only exception of the scaling factor for fission barriers, for all other parameters in our calculations we used standard values recommended in Ref. [8], the most important of them are explicitly mentioned below. We assume that the ER formation proceeds via two independent steps: complete fusion of projectile and target nuclei to form a compound nucleus, followed by its decay via the competition between nuclear fission, particle evaporation, and $\gamma$ decay. The fusion cross section was calculated within a one-dimensional barrier penetration model [41-43] which uses the partial wave summation according to the expression

$$
\sigma_{\mathrm{fus}}(E)=\pi \lambda^{2} \sum_{l=0}^{l_{\mathrm{cr}}}(2 l+1) T_{l}(E),
$$

where $\lambda$ is the entrance channel's reduced wavelength and $T_{l}(E)$ are the transmission coefficients for the penetrating waves. The sum over the angular momentum quantum 
number $l$ is limited to a relatively small range below the critical value $l_{\text {cr }}$ given by the rapid decrease of the survival probability with angular momentum. Some typical values in this region of nuclei are $l_{\mathrm{cr}} \sim 30$ for mercury isotopes, $l_{\mathrm{cr}} \sim 25$ for bismuth and polonium isotopes, and $l_{\mathrm{cr}} \leqslant 20$ for radium isotopes; see, e.g. [9-11] and references therein.

Two different models were used for the $T_{l}(E)$ values in our study. In the simplest approach, denoted further as inverted parabola (IP), the Coulomb plus nuclear part of the barrier for each partial wave can be approximated near its maximum by an inverted harmonic oscillator potential of height $B_{l}$ and curvature $\hbar \omega_{l}$ (see [42,43] and references therein). For such a parametrization, the transmission coefficient $T_{l}(E)$ for each partial wave is given by the Hill-Wheeler formula $T_{l}(E)=\left\{1+\exp \left[2 \pi\left(B_{l}-E\right) / \hbar \omega_{l}\right]\right\}^{-1}$ [44]. This approach is known to work well for most complete fusion reactions at energies above the empirical Bass barrier [45], while the subbarrier fusion in the reactions with heavy projectiles is strongly underestimated, see discussion in Refs. $[42,43]$.

An alternative variant to calculate the $T_{l}(E)$ values was also considered with the aim of accounting for the subbarrier fusion observed in most of the studied reactions. Subbarrier fusion is a complex phenomenon, and for detailed discussion of different processes (e.g., channel coupling, transfer and inelastic couplings, static and dynamic deformation) leading to cross section enhancement, we refer to reviews [43]. In our study, we exploited a phenomenological model that involves the barrier fluctuations (BF) with an amplitude correlated with the collective surface properties of the colliding nuclei $[9,41,43]$. As discussed in the literature, this rather simple model allows a surprisingly good description of the fusion excitation functions over the full energy region in terms of only two parameters: the average barrier height $B_{0}$ and its standard (Gaussian) deviation $\sigma\left(B_{0}\right)$. A relevant example of the application of this approach to a number of nearly symmetrical reactions induced by ${ }^{100} \mathrm{Mo}$ and ${ }^{96} \mathrm{Zr}$ projectiles is discussed in Ref. [9]. Anticipating the discussion below, we note that above the Bass barrier both models give similar calculated cross sections with large differences arising only below the barrier.

For the deexcitation stage, the HIVAP code uses standard evaporation theory by considering the competition between neutron, proton, and $\alpha$ and $\gamma$ decays, as well as fission [8]. The nuclear level density parameters (in fact, their ratio $\tilde{a}_{f} / \tilde{a}_{v}$ ) and fission barriers are the two most important ingredients in such calculations. For the former, we used the Reisdorf parametrization from Ref. [8], while the excitation energy dependence of the level density was considered following Ignatuk's prescription. A constant of $E_{D}=18.5 \mathrm{MeV}$ was used to account for the damping of the shell effects in the level density $[8,46]$. The small shell effects at the saddle point were neglected, while the ground state shell corrections $\Delta W_{\mathrm{gs}}$ were calculated as the difference between empirical masses taken from Ref. [47] and LD masses from the model of Cohen, Plasil, and Swiatecki [1].

The fission barriers $B_{f}(1)$ were calculated with the formula: $B_{f}(\mathrm{l})=C_{f} \times B_{f}^{\mathrm{LD}}(\mathrm{l})+\Delta W^{\exp }$, where $C_{f}$ is a free scaling parameter, $B_{f}^{\mathrm{LD}}(\mathrm{l})$ is the fission barrier of the rotating $\mathrm{LD}$ model [1], and $\Delta W^{\text {exp }}$ is a (negative) correction to the fission

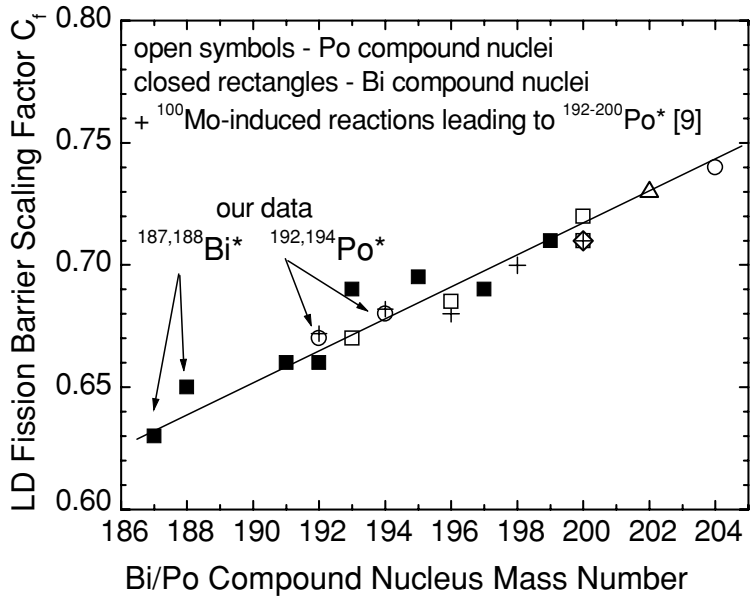

FIG. 4. LD fission barrier scaling factor $C_{f}$ as a function of the $\mathrm{Bi}$ or Po compound nucleus mass number. See text for more details and for the list of reactions. Only above-barrier evaporation channels were considered in the comparison of the experimental and calculated cross sections. The solid line is given to guide the eye.

barrier which is usually taken as being equal to the ground state shell correction $\Delta W_{\mathrm{gs}}$. In this way, the scaling factor $C_{f}$ was the only adjustable parameter in our calculations to reproduce the maxima of the excitation functions of the $x n$ and pxn evaporation channels.

\section{B. Reactions leading to polonium compound nuclei}

\section{Calculations for the previously studied reactions}

As a first step in our analysis, to check the consistency of our calculations compared to earlier studies [10], we performed HIVAP calculations for the following previously studied reactions leading to the polonium compound nuclei ${ }^{192-204} \mathrm{Po}^{*}:{ }^{100} \mathrm{Mo}+{ }^{92-100} \mathrm{Mo} \rightarrow{ }^{192-200} \mathrm{Po}^{*}$ [9], ${ }^{36} \mathrm{Ar}+{ }^{160} \mathrm{Dy} \rightarrow{ }^{196} \mathrm{Po}^{*} \quad[36],{ }^{40} \mathrm{Ar}+{ }^{160} \mathrm{Dy} \rightarrow{ }^{200} \mathrm{Po}^{*}{ }^{84} \mathrm{Kr}+$ ${ }^{116} \mathrm{Cd} \rightarrow{ }^{200} \mathrm{Po}^{*}[17],{ }^{31} \mathrm{P}+{ }^{169} \mathrm{Tm} \rightarrow{ }^{200} \mathrm{Po}^{*},{ }^{27} \mathrm{Al}+{ }^{175} \mathrm{Lu} \rightarrow$ ${ }^{202} \mathrm{Po}^{*}$ [10], ${ }^{20} \mathrm{Ne}+{ }^{184} \mathrm{~W} \rightarrow{ }^{204} \mathrm{Po}^{*}$, and ${ }^{40} \mathrm{Ar}+{ }^{164} \mathrm{Dy} \rightarrow$ ${ }^{204} \mathrm{Po}^{*}$ [16]. From these calculations, we derived an important conclusion that for all the cases for energies above the respective Bass barriers, a satisfactory agreement (within $50 \%$ ) between the calculated and experimental data could be obtained by gradually reducing the scaling parameter $C_{f}$ from 0.74(1) for ${ }^{204} \mathrm{Po}^{*}$ down to 0.67 (1) for ${ }^{192} \mathrm{Po}^{*}$. Figure 4 shows the deduced dependence of the $C_{f}$ value on the polonium mass number for different reactions, including those studied in this work (to be discussed in the following sections). The uncertainty of 0.01 reflects the $C_{f}$ range for different reactions leading to the same $\mathrm{CN}$ and corresponds to the change of the calculated cross section by about $30 \%$, which is within the typical uncertainty of the absolute cross section measurements $(\sim 40 \%)$. Also, the shape and relative position of the excitation functions on the energy axis were reproduced quite well. As one example relevant to our work, Fig. 5 demonstrates the good description of the above-barrier $2 n-3 n$ and $p 1 n-p 5 n$ 


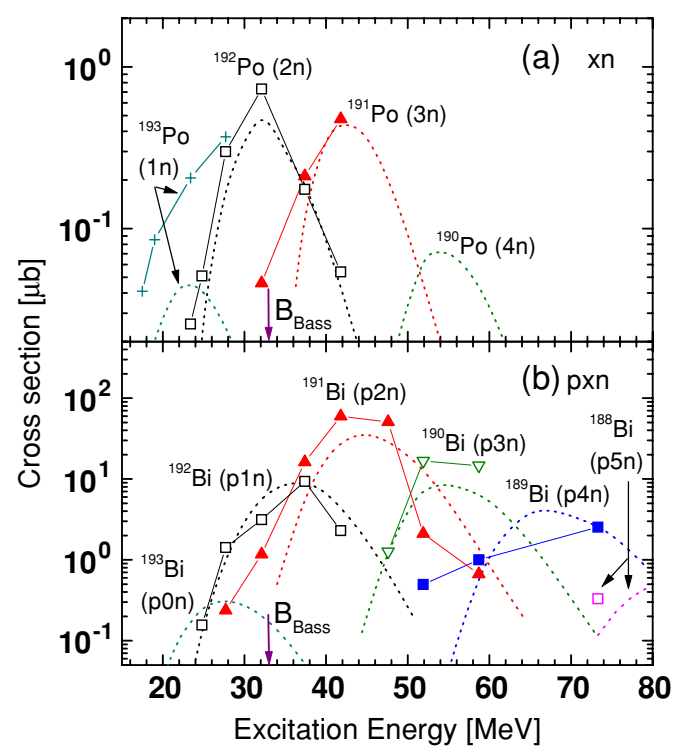

FIG. 5. (Color online) Comparison of calculated (dotted lines) and measured (solid lines with symbols) cross sections for the ${ }^{100} \mathrm{Mo}+{ }^{94} \mathrm{Mo} \rightarrow{ }^{194} \mathrm{Po}^{*}$ reaction: (a) $x n$ channel; (b) pxn channel. Experimental data are from [9]. Scaling factor $C_{f}=0.68$ and $\mathrm{BF}$ approach were used in the calculations.

evaporation channels of the ${ }^{100} \mathrm{Mo}+{ }^{94} \mathrm{Mo} \rightarrow{ }^{194} \mathrm{Po}^{*}$ reaction studied in Ref. [9]. The same compound nucleus is produced in the ${ }^{52} \mathrm{Cr}+{ }^{142} \mathrm{Nd} \rightarrow{ }^{194} \mathrm{Po}^{*}$ reaction studied in this work. A comparative analysis of these two reactions will be given in the next section.

We also note that the use of the fission barriers according to the Sierk model [2] in our calculations leads to a very similar trend of the scaling factor $C_{f}$ as in Fig. 4, but the absolute values are higher by about 0.05 . This naturally reflects the fact that in this mass region, the fission barriers of [2] are lower by about $5 \%$ than those from the LD model of [1]. In fact, the development of the Sierk model itself was partly motivated by the persistently lower experimental fission barriers compared to the RLDM predictions, see discussion in [2].

It is important to stress that the $C_{f}=0.71(1)$ value obtained in our calculations for the ${ }^{31} \mathrm{P}+{ }^{169} \mathrm{Tm} \rightarrow{ }^{200} \mathrm{Po}^{*}$ and ${ }^{100} \mathrm{Mo}+{ }^{100} \mathrm{Mo} \rightarrow{ }^{200} \mathrm{Po}^{*}$ reactions agrees with the value of $C_{f}=0.73$ derived in Ref. [10] by using the ALICE code for the same reactions. This shows that with the proper application of different statistical model codes, the extracted results and parameters do not depend on the code and are consistent with each other. Furthermore, Fig. 4 shows that the earlier conclusion of study [10] that the different reactions leading to the $\mathrm{CN}^{200} \mathrm{Po}^{*}$ require practically the same value of $C_{f}$ independently of the mass asymmetry in the entrance channel can now be generalized to a broader region of $\mathrm{CN}\left({ }^{196-204} \mathrm{Po}^{*}\right)$. The above data and conclusions give us confidence and basis for the calculations for even more neutron-deficient isotopes ${ }^{190,192,194}$ Po, studied in our experiments at SHIP. The results of the HIVAP calculations are shown in Figs. 1-3 by the dashed lines (IP variant) and dotted lines (BF variant).

\section{2. ${ }^{52} \mathrm{Cr}+{ }^{142} \mathrm{Nd} \rightarrow{ }^{194} \mathrm{Po}^{*}$ data and comparison with the ${ }^{100} \mathrm{Mo}+{ }^{94} \mathrm{Mo} \rightarrow{ }^{194} \mathrm{Po}^{*}$ reaction}

We now consider in detail the ${ }^{52} \mathrm{Cr}+{ }^{142} \mathrm{Nd} \rightarrow{ }^{194} \mathrm{Po}^{*}$ reaction, for which the most comprehensive data set was collected in our experiments. Figure 1 shows that above the Bass barrier, despite the cross section variation of more than five orders of magnitude from $130(30) \mu \mathrm{b}\left({ }^{192} \mathrm{Bi}\right)$ down to $0.6(3) \mathrm{nb}\left({ }^{188} \mathrm{Po}\right)$, the IP variant of the calculations describes the data quite accurately by using the scaling factor of $C_{f}(\mathrm{LD})=0.68$ (or $C_{f}=0.73$ if the barriers from Ref. [2] are used, see above). This value follows well the decreasing trend of the $C_{f}$ parameter as a function of the neutron deficit of Po compound nuclei (Fig. 4). Importantly, as in the case of the ${ }^{100} \mathrm{Mo}+{ }^{94} \mathrm{Mo} \rightarrow{ }^{194} \mathrm{Po}^{*}$ reaction (see Fig. 5) a good agreement between the experimental and calculated values could be obtained simultaneously for the $x n$ and pxn channels, up to the $p 6 n$ channel $\left({ }^{187} \mathrm{Bi}\right)$. This proves that both neutron and proton emissions are taken into account properly in our calculations. We also note a good correspondence between the calculated and measured shapes and positions on the energy axis for the $x n$ and pxn excitation functions, both for the ${ }^{52} \mathrm{Cr}+{ }^{142} \mathrm{Nd}$ and ${ }^{100} \mathrm{Mo}+{ }^{94} \mathrm{Mo}$ reactions and the fact that the measured maxima of the respective channels are observed at the same excitation energy for both reactions. This suggests that there are no significant deficiencies related to the nuclear masses and/or calculated thermodynamical properties of the emitted particles (e.g., emission energies).

Special attention was paid in these measurements to the energy region in the vicinity of the Bass barrier, which corresponds to an excitation energy of $E^{* \text { Bass }}=33 \mathrm{MeV}$. This relatively low excitation energy at the barrier reflects the fact that both ${ }^{52} \mathrm{Cr}$ and ${ }^{142} \mathrm{Nd}$ are semimagic spherical nuclei, having $N=28$ and $N=82$ closed shells, respectively. Both measurements at $E^{*}=32$ and $35 \mathrm{MeV}$ resulted in high yields for the $2 n\left({ }^{192} \mathrm{Po}\right)$ and $p 1 n\left({ }^{192} \mathrm{Bi}\right)$ channels (see Fig. 1 of Ref. [25] for the data at $E^{*}=32 \mathrm{MeV}$ ), therefore their cross sections were deduced with sufficient accuracy. Figure 1 clearly demonstrates that in the vicinity of the Bass barrier, the calculated cross sections for ${ }^{192} \mathrm{Po}$ and ${ }^{192} \mathrm{Bi}$ are suppressed by at least two orders of magnitude in the IP approach.

In contrast, near and below the barrier, the calculations within the BF approach (shown by the dotted lines in Fig. 1) result in considerably enhanced cross sections, which are in good agreement [for $\sigma\left(B_{0}\right)=4 \%$ ] with the experimental data, both for the ${ }^{192} \mathrm{Po}$ and ${ }^{192} \mathrm{Bi}$ nuclei. For the sake of clarity, the calculated data for this variant are shown only for ${ }^{191,192} \mathrm{Bi}$ and ${ }^{191,192} \mathrm{Po}$. As expected, above the barrier, both the IP and BF approaches give very similar calculated cross sections as exemplified by the data for ${ }^{191} \mathrm{Bi}(p 2 n)$ and ${ }^{191} \mathrm{Po}(3 n)$. The chosen value of $\sigma\left(B_{0}\right)$ is within the range of $3-5 \%$ recommended in the literature for the reactions involving spherical partners $[7,41]$. Owing to the scarcity of our data in the subbarrier region, we did not aim at an exact reproduction of the experimental data, rather we wanted to show the trend of the calculated cross sections in cases where static and/or dynamic effects are considered (though phenomenologically). As an example, we found that the change of standard barrier deviation within the range of 
$\sigma\left(B_{0}\right)=3-5 \%$ influences somewhat the energy dependence below the barrier, but does not significantly change the absolute cross section values.

In our opinion, from the cross section point of view, the ${ }^{52} \mathrm{Cr}+{ }^{142} \mathrm{Nd} \rightarrow{ }^{194} \mathrm{Po}$ * reaction is one of the best to study the isotopes ${ }^{191,192} \mathrm{Po}\left[\sigma\left({ }^{191} \mathrm{Po}\right)=1.6(4) \mu \mathrm{b}, \sigma\left({ }^{192} \mathrm{Po}\right)=8(2) \mu \mathrm{b}\right.$, see Table III). Furthermore, because of a relatively low excitation energy at beam energies corresponding to the maxima of ${ }^{191,192}$ Po, only a few competing pxn channels are open, which strongly reduces background from other isotopes, e.g., from the $\alpha x n$ channel or channels involving evaporation of two charged particles. The ${ }^{36} \mathrm{Ar}+{ }^{160} \mathrm{Dy} \rightarrow{ }^{192} \mathrm{Po}+4 n$ reaction, studied in Ref. [36], has a comparable cross section of about 10 $\mu \mathrm{b}$, but this reaction suffers from the relatively low enrichment of ${ }^{160} \mathrm{Dy}(\sim 70 \%)$ currently available. The latter results in much larger amounts of the heavier $\mathrm{Bi}$ and Po isotopes, produced on the admixtures of the heavier Dy isotopes in the target, than of ${ }^{192} \mathrm{Po}$ itself. This advantage of the ${ }^{52} \mathrm{Cr}$-induced reaction is clearly seen by comparing the $\alpha$ spectra in Fig. 1 of Ref. [25,36].

To conclude, the fact that the same value of $C_{f}=0.68$ is required for a good description of the $x n$ and pxn channels of both the ${ }^{52} \mathrm{Cr}+{ }^{142} \mathrm{Nd} \rightarrow{ }^{194} \mathrm{Po}^{*}$ and ${ }^{100} \mathrm{Mo}+{ }^{94} \mathrm{Mo} \rightarrow{ }^{194} \mathrm{Po}^{*}$ reactions proves that, as for the heavier polonium isotopes and in agreement with the compound nucleus formation hypothesis, the production and deexcitation of the compound nucleus ${ }^{194} \mathrm{Po}^{*}$ do not depend on the reaction (apart from the trivial $\pi \lambda^{2}$ dependence) up to nearly complete symmetry in the entrance channel. Above the barrier, due to the $\pi \lambda^{2}$ dependence, the cross sections are lower in the ${ }^{100} \mathrm{Mo}+{ }^{94} \mathrm{Mo} \rightarrow{ }^{194} \mathrm{Po}^{*}$ reaction than in the ${ }^{52} \mathrm{Cr}+{ }^{142} \mathrm{Nd} \rightarrow{ }^{194} \mathrm{Po}^{*}$ reaction.

\section{3. ${ }^{50} \mathrm{Cr}+{ }^{142} \mathrm{Nd} \rightarrow{ }^{192} \mathrm{Po}^{*}$ data and comparison with the ${ }^{100} \mathrm{Mo}+{ }^{92} \mathrm{Mo} \rightarrow{ }^{192} \mathrm{Po}^{*}$ reaction}

A similar analysis was performed for our data from the ${ }^{50} \mathrm{Cr}+{ }^{142} \mathrm{Nd} \rightarrow{ }^{192} \mathrm{Po}^{*}$ reaction. Figure 2 shows that by using the scaling factor $C_{f}=0.67$, we achieved a good agreement between the calculated and measured data for the $x n$ and $p x n$ channels of this reaction, both in terms of the absolute values and energy dependence of the cross sections. Both the IP and $\mathrm{BF}$ variants of the calculations resulted in similar values above the barrier as shown for ${ }^{189} \mathrm{Bi}$ and ${ }^{189} \mathrm{Po}$. The subbarrier cross section is strongly enhanced in the $\mathrm{BF}$ approach, though the enhancement is somewhat lower than in the ${ }^{52} \mathrm{Cr}+{ }^{142} \mathrm{Nd}$ reaction due to the larger excitation energy at the barrier for the ${ }^{50} \mathrm{Cr}$-induced reaction $\left(E^{*_{\text {Bass }}}=36 \mathrm{MeV}\right)$. This explains why the $2 n$ channel $\left({ }^{190} \mathrm{Po}\right)$ has a somewhat lower calculated cross section than the $3 n$ channel $\left({ }^{189} \mathrm{Po}\right)$. Although we did not measure the data at the maximum of ${ }^{189} \mathrm{Po}$, the calculations, along with the data measured at $E^{*}=55 \mathrm{MeV}$, strongly suggest that this reaction is one of the best to produce the isotope ${ }^{189} \mathrm{Po}$ : the expected maximum cross section is $\sigma_{\text {calc }}(3 n) \sim 230 \mathrm{nb}$ (see also Table III), which should be compared with a value of $\sigma_{\text {exp, } \max }\left({ }^{189} \mathrm{Po}, 5 n\right)=30(10) \mathrm{nb}$, measured in the ${ }^{52} \mathrm{Cr}+{ }^{142} \mathrm{Nd}$ reaction [Fig. 1(a)].

Similar conclusions were derived for the previously measured data from the ${ }^{100} \mathrm{Mo}+{ }^{92} \mathrm{Mo} \rightarrow{ }^{192} \mathrm{Po}^{*}$ reaction in
Ref. [9], for which the same scaling factor was used in our calculations, see Fig. 4. We note that given the scarcity of the data for the $x n$ channel of ${ }^{192} \mathrm{Po}^{*}$ in Ref. [9], mostly pxn channels above the Bass barrier were considered in the comparison. As expected, because of the $\pi \lambda^{2}$ dependence, the cross sections are systematically lower in the ${ }^{100} \mathrm{Mo}+{ }^{92} \mathrm{Mo}$ reaction than in the ${ }^{50} \mathrm{Cr}+{ }^{142} \mathrm{Nd}$ reaction. However, the fact that the same value of $C_{f}=0.67$ was used in both cases implies that the production and deexcitation of $\mathrm{CN}^{192} \mathrm{Po}^{*}$ do not depend on the asymmetry in the entrance channel.

\section{The ${ }^{98} \mathrm{Mo}+{ }^{92} \mathrm{Mo} \rightarrow{ }^{190} \mathrm{Po}^{*}$ reaction}

By moving to the more neutron-deficient compound nucleus ${ }^{190} \mathrm{Po}^{*}$, the $x n$ and $p x n$ evaporation cross sections are expected to decrease to the subnanobarn and sub microbarn regions, respectively.

Figure 3 shows the relative production cross sections for the $p 1 n-p 3 n$ channels measured in the ${ }^{98} \mathrm{Mo}+{ }^{92} \mathrm{Mo} \rightarrow$ ${ }^{190} \mathrm{Po}^{*}$ reaction. The extraction of the absolute values in this experiment was hampered by the relatively low yield, by the complexity of the $\alpha$ spectra due to admixtures of the heavier Mo isotopes in the target, and by a possible admixture of the ${ }^{97}$ Mo isotope in the main ${ }^{98}$ Mo beam (see also the next section). Therefore, only a crude estimate of $\sigma\left({ }^{187} \mathrm{Bi}\right)$ of a few hundred nanobarns could be extracted from the data.

To show the trend of the calculated cross sections, we provide in Fig. 3 the results obtained with the BF approach and normalized to the maximum experimental cross section of ${ }^{187} \mathrm{Bi}$. One can see that due to the use of the semimagic target isotope ${ }^{92} \mathrm{Mo}(N=50)$ and the more symmetric reaction, the excitation energy $E_{\text {Bass }}^{*}=28.2 \mathrm{MeV}$ at the Bass barrier of the ${ }^{98} \mathrm{Mo}+{ }^{92} \mathrm{Mo}$ reaction is about $5 \mathrm{MeV}$ lower than that for the ${ }^{100} \mathrm{Mo}+{ }^{94} \mathrm{Mo}$ reaction (see Fig. 5). This explains the less suppressed cross section for the $p 1 n$ channel of the former reaction.

\section{5. $T h e^{46} \mathrm{Ti}+{ }^{144} \mathrm{Sm} \rightarrow{ }^{190} \mathrm{Po}^{*}$ reaction}

For the sake of completeness, in Table III we also provide preliminary cross section values for the new isotopes ${ }^{186,187}$ Po, produced recently at SHIP in, respectively, the $4 n$ and $3 n$ evaporation channels of the complete fusion reaction ${ }^{46} \mathrm{Ti}+{ }^{144} \mathrm{Sm} \rightarrow{ }^{190} \mathrm{Po}^{*}$. The detailed analysis of the cross section data for ${ }^{186,187} \mathrm{Po}$ and ${ }^{185-188} \mathrm{Bi}$ from this reaction will be presented elsewhere [27].

\section{General conclusions for ${ }^{190-204} \mathrm{Po}^{*}$ compound nuclei and optimal reactions leading to the isotopes ${ }^{186-192}$ Po}

This subsection summarizes our conclusions for the isotopes ${ }^{186-192}$ Po.

(1) Above the barrier, the production and deexcitation of the ${ }^{192-204}{ }^{2}{ }^{*}$ compound nuclei do not depend on the reaction (apart from the trivial $\pi \lambda^{2}$ dependence) up to nearly complete symmetry in the entrance channel. 
(2) Above the barrier, satisfactory agreement between experimental and calculated data could be obtained for all reactions, except for ${ }^{98} \mathrm{Mo}+{ }^{92} \mathrm{Mo}$ (see the reasons above). Simultaneous agreement for the $x n$ and $p x n$ channels was achieved by varying only one adjustable parameter, the scaling factor $C_{f}$ of the rotating LD model. A gradual reduction of the $\mathrm{LD}$ fission barrier is required from $0.74(1)$ for ${ }^{204} \mathrm{Po}^{*}$ up to $0.67(1)$ for ${ }^{192} \mathrm{Po}^{*}$.

(3) In the case of sub- or near-barrier reactions, the $\mathrm{BF}$ variant of calculations provides much enhanced cross section values, which are in a better agreement with the experimental data than the IP variant values.

(4) A rapid increase of the ratio $\sigma(p x n) / \sigma(x n)$ with the increasing neutron deficit of polonium compound nuclei is clearly seen from the measured data. For comparison, the cross section ratio for the above-barrier $p 2 n$ and $3 n$ channels of the ${ }^{100} \mathrm{Mo}+{ }^{96} \mathrm{Mo} \rightarrow{ }^{196} \mathrm{Po}^{*}$ reaction is $\sigma(p 2 n) / \sigma(3 \mathrm{n}) \sim 1$ [9], while for the ${ }^{52} \mathrm{Cr}+{ }^{142} \mathrm{Nd} \rightarrow$ ${ }^{194} \mathrm{Po}^{*}$ and ${ }^{100} \mathrm{Mo}+{ }^{94} \mathrm{Mo} \rightarrow{ }^{194} \mathrm{Po}^{*}$ reactions, the ratios are $\sigma(p 2 n) / \sigma(3 n) \sim 100$ and $\sim 120$, respectively (cf. Figs. 1 and 5). The ratio $\sigma(p 2 n) / \sigma(3 n) \sim 250$ was deduced for the ${ }^{46} \mathrm{Ti}+{ }^{144} \mathrm{Sm} \rightarrow{ }^{190} \mathrm{Po}^{*}$ reaction in Ref. [27]. This is due to the increase of the neutron binding energies along with the decrease of the proton binding energies for more neutron-deficient nuclei, which makes the proton emission preferential. Furthermore, at the same neutron deficit, the survival probability of Po nuclei is lower than that of $\mathrm{Bi}$ isotopes because of the larger fission barriers of the latter nuclei.

(5) It is important to stress the drop in the cross section value for ${ }^{192} \mathrm{Po}$ when it is produced in the pxn channel, rather than in the $x n$ channel. To give an example, we added to Table III the cross section value for ${ }^{192}$ Po produced in the $p 2 n$ channel of the ${ }^{51} \mathrm{~V}+{ }^{144} \mathrm{Sm} \rightarrow{ }^{195} \mathrm{At}^{*}$ reaction recently studied at SHIP [37]. The measured value $\sigma\left({ }^{192} \mathrm{Po}\right)=$ $0.8(2) \mu \mathrm{b}$ is a factor of 10 lower than when ${ }^{192} \mathrm{Po}$ is produced in the $2 n$ or $4 n$ channels. This will also apply to other neutron-deficient Po isotopes, and it is explained by the higher fissility of the At compound nuclei compared to Po compound nuclei at the same or similar neutron number.

(6) In Table III, along with the measured data, we also provide the HIVAP predictions for some prospective reactions leading to ${ }^{188-192} \mathrm{Po}$, for which the $C_{f}$ value was chosen according to the trend in Fig. 4. As expected, the highest cross section value for a particular nucleus is achieved when it is produced in the above-barrier $x n$ channel of an asymmetric reaction with the lowest number of emitted neutrons.

\section{Reactions leading to Bi compound nuclei}

\section{Calculations for the previously studied reactions}

To check the consistency of our calculations compared to the earlier works, we performed HIVAP calculations for the following previously studied reactions leading to the bismuth compound nuclei ${ }^{191-199} \mathrm{Bi}^{*}:{ }^{40} \mathrm{Ar}+{ }^{159} \mathrm{~Tb} \rightarrow{ }^{199} \mathrm{Bi}^{*}$ [11],

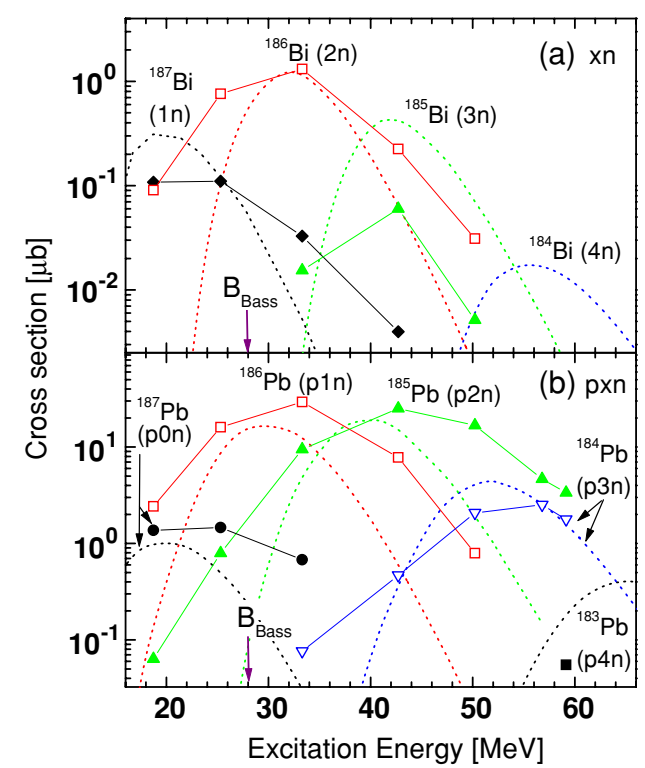

FIG. 6. (Color online) Comparison of calculated (dotted lines) and measured (solid lines with symbols) cross sections for ${ }^{95} \mathrm{Mo}+{ }^{93} \mathrm{Nb} \rightarrow{ }^{188} \mathrm{Bi}^{*}$ reaction: (a) $x n$ channel; (b) pxn channel. Scaling factor $C_{f}=0.65$ and $\mathrm{BF}$ approach were used in calculations.

${ }^{32} \mathrm{~S}+{ }^{165} \mathrm{Ho} \rightarrow{ }^{197} \mathrm{Bi}^{*} \quad$ [48], ${ }^{86} \mathrm{Kr}+{ }^{109} \mathrm{Ag} \rightarrow{ }^{195} \mathrm{Bi}^{*} \quad$ [49], ${ }^{83} \mathrm{Kr}+{ }^{109} \mathrm{Ag} \rightarrow{ }^{192} \mathrm{Bi}^{*} \quad[50], \quad{ }^{40} \mathrm{Ca}+{ }^{151,153} \mathrm{Eu} \rightarrow{ }^{191,193} \mathrm{Bi}^{*}$ [11]. As in the case of polonium compound nuclei, satisfactory agreement between the calculated and measured cross sections was obtained by gradually reducing the scaling factor from $C_{f}=0.71$ for ${ }^{199} \mathrm{Bi}^{*}$ down to $C_{f}=0.66$ for ${ }^{191} \mathrm{Bi}^{*}$ (Fig. 4). These values of $C_{f}$ follow the same trend as deduced for compound nuclei ${ }^{191,193,199} \mathrm{Bi}^{*}$ in the study [11] by using the ALICE statistical model code.

\section{The ${ }^{95} \mathrm{Mo}+{ }^{93} \mathrm{Nb} \rightarrow{ }^{188} \mathrm{Bi}^{*}$ reaction}

Figure 6 shows the $x n$ and pxn cross sections measured in the ${ }^{95} \mathrm{Mo}+{ }^{93} \mathrm{Nb} \rightarrow{ }^{188} \mathrm{Bi}^{*}$ reaction along with the values calculated using the scaling factor $C_{f}=0.65$ and the $\mathrm{BF}$ approach. As for other reactions discussed above, a satisfactory description of the $1 n-2 n$ and $p 0 n-p 3 n$ channels was achieved, with the exception of the $3 n$ channel $\left({ }^{185} \mathrm{Bi}\right)$, for which the calculations overestimate the maximum of the excitation function by about seven times. This could indicate a much steeper decrease of the experimental $x n$ cross sections compared to the calculations, but the good description of the $3 n$ and $p 2 n$ channels of the ${ }^{94} \mathrm{Mo}+{ }^{93} \mathrm{Nb} \rightarrow{ }^{187} \mathrm{Bi}^{*}$ reaction (see next section) disagrees with this interpretation. The reason for this discrepancy is not clear at the moment. The measured cross section of the proton emitter ${ }^{185} \mathrm{Bi}$ is $\sigma(3 n)=50(15)$ $\mathrm{nb}$, thus this reaction is one of the best for studying this nucleus. As discussed in our recent work [31], the alternative reaction ${ }^{95} \mathrm{Mo}\left({ }^{92} \mathrm{Mo}, p n\right){ }^{185} \mathrm{Bi}$ used in the studies [51] has a lower cross section of about 4-6 nb. This is due to a combination of two reasons. First of all, the $p 1 n$ channel of the Mo+Mo reactions is usually somewhat sub barrier as seen, 
for example, for ${ }^{192} \mathrm{Bi}$ produced in the $p 1 n$ channel of the ${ }^{100} \mathrm{Mo}+{ }^{94}$ Mo reaction (see Fig. 5 and data from Ref. [9]). Secondly, the polonium compound nuclei have higher fissility than the bismuth compound nuclei at the comparable neutron deficit and excitation energy.

We note that the experimental excitation functions are much broader than the calculated ones, which is in contrast with the aforementioned more asymmetric reactions leading to the heavier Bi and Po compound nuclei. Two reasons could cause this effect. First of all, we used a ${ }^{93} \mathrm{Nb}$ target with a thickness of $900(50) \mu \mathrm{g} / \mathrm{cm}^{2}$, which is substantially thicker than the targets used in other experiments (cf. Table I). Clearly, the larger beam energy spread in the target results in the broadening of the experimental excitation functions. Secondly, natural Mo material was used in the ion source of the UNILAC accelerator which resulted in some difficulties in completely separating the main ${ }^{95}$ Mo beam ( $15.9 \%$ natural abundance) from the neighboring ${ }^{94,96}$ Mo isotopes with $9.25 \%$ and $16.7 \%$ natural abundance, respectively. Such an admixture of the neighboring isotopes in the beam will broaden the excitation functions, but the maximum, corresponding to the proper beam energy of ${ }^{95} \mathrm{Mo}$, should not be influenced. Therefore, the maxima of the excitation functions in Fig. 6 should be reliable.

\section{3. $T h e{ }^{94} \mathrm{Mo}+{ }^{93} \mathrm{Nb} \rightarrow{ }^{187} \mathrm{Bi}^{*}$ reaction}

Cross sections for the $2 n-3 n$ and $p 1 n-p 3 n$ channels of the ${ }^{94} \mathrm{Mo}+{ }^{93} \mathrm{Nb} \rightarrow{ }^{187} \mathrm{Bi}^{*}$ reaction are compared with calculations in Fig. 7. With the exception of the near-barrier $p 1 n$

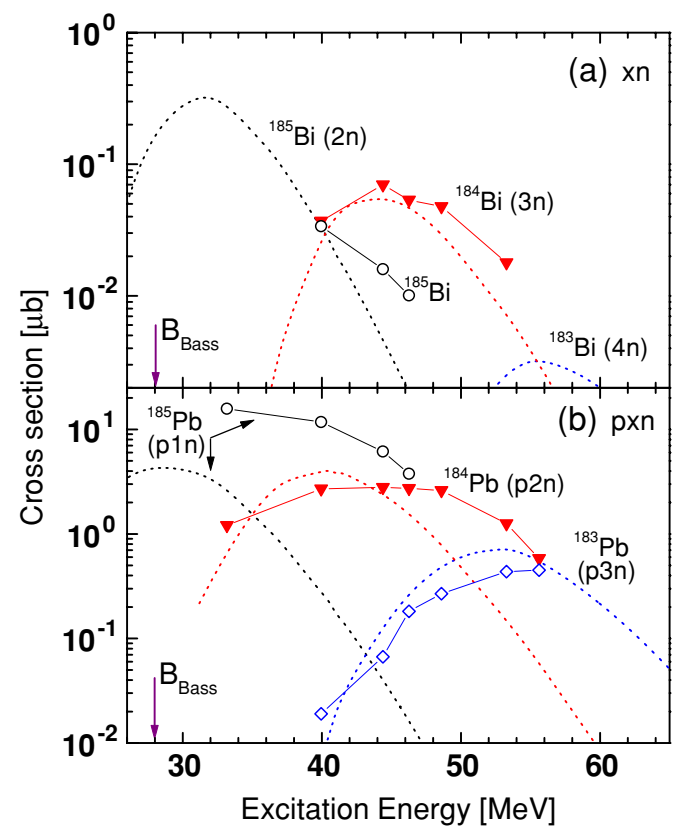

FIG. 7. (Color online) Comparison of calculated (dotted lines) and measured (solid lines with symbols) cross sections for the ${ }^{94} \mathrm{Mo}+{ }^{93} \mathrm{Nb} \rightarrow{ }^{187} \mathrm{Bi}^{*}$ reaction. (a) $x n$ channel; (b) $p x n$ channel. The scaling factor $C_{f}=0.63$ and the $\mathrm{BF}$ approach were used in the calculations. channel $\left({ }^{185} \mathrm{~Pb}\right)$, a satisfactory description of the data, by using $C_{f}=0.63$ and the BF approach, was obtained. We note that the large uncertainty in the $\alpha$-branching ratio $b_{\alpha}=34(25) \%$ of ${ }^{185} \mathrm{~Pb}$ [26] could be partially responsible for the discrepancy for this nucleus. A possible admixture of ${ }^{95} \mathrm{Mo}$ in the main ${ }^{94}$ Mo beam could also be the reason for the broadening of the experimental excitation functions.

It is interesting to note that both HIVAP calculations and the partial excitation function measured for ${ }^{185} \mathrm{Bi}$ [see Fig. 7(a)] suggest that its cross section in this reaction could be a few times higher than in the ${ }^{95} \mathrm{Mo}+{ }^{93} \mathrm{Nb}$ reaction (cf. Figs. 6 and 7). Unfortunately, because of the large background from the scattered projectiles, no such measurement could be made during our experiment to check this conclusion.

To conclude, the analysis of the ${ }^{94,95} \mathrm{Mo}+{ }^{93} \mathrm{Nb} \rightarrow$ ${ }^{187,188} \mathrm{Bi}^{*}$ reactions studied in this work together with the data for the previously studied reactions leading to 191-193,195,197,199 $\mathrm{Bi}$ compound nuclei showed that above the barrier, satisfactory agreement between experimental and calculated cross sections could be obtained simultaneously for the $x n$ and $p x n$ channels by varying only one adjustable parameter-the scaling factor $C_{f}$ of the rotating LD model. A gradual reduction of the LD fission barrier is required by changing the $C_{f}$ value from 0.71 for ${ }^{199} \mathrm{Bi}^{*}$ down to 0.63 for ${ }^{187} \mathrm{Bi}^{*}$.

\section{CONCLUSIONS}

Absolute cross sections of the $x n$ and pxn evaporation channels for the very neutron-deficient ${ }^{187,188} \mathrm{Bi}^{*}$ and ${ }^{190,192,194} \mathrm{Po}^{*}$ compound nuclei have been studied by using heavy-ioninduced complete fusion reactions in a series of experiments at the velocity filter SHIP. The results, together with the previously known data for the heavier $\mathrm{Bi}$ and Po nuclides, were compared with the results of statistical model calculations carried out with the HIVAP code.

It is shown that a satisfactory description of the experimental data in the long chains of $\mathrm{Po}$ and $\mathrm{Bi}$ isotopes requires a significant (up to $35 \%$ ) reduction of the theoretical fission barriers. Very similar behavior of the scaling factor $C_{f}$ as a function of the neutron deficit of the Bi and Po nuclei (Fig. 4) suggests that the underlying mechanism in the reduction of the LD fission barriers is the same in both cases. We note that already in 1978 Beckerman and Blann [3] pointed out the necessity of reducing the LD fission barrier in the calculations in order to describe the fission cross section data correctly for the neutron-deficient nuclei in the region between $\mathrm{Pd}$ and Os. At that time, given the lack of reliable experimental data for the heavier systems, the conclusion was that this effect is specific to the lighter systems only, which was later reflected in the Sierk model [2]. The new experimental data discussed in this work, together with the previously known data in the $\mathrm{Pb}-\mathrm{U}$ region $[6-13,15]$ suggest that the reduction of the LD fission barriers is a more general phenomenon, and one has to search for a new approach to explain the extended data set. One such attempt was undertaken in study [52], which suggested a new formula for the isospin dependence of the LD fission 
barriers. From the point of view of the analysis, it also became clear that the new degrees of freedom should be included, one of them being the pre/post-scission neutron multiplicities, see discussion in $[6,14]$ and references therein.

Our data suggest that, mainly due to strongly increased fissility above the shell closure at $Z=82$, the production of very neutron-deficient $\mathrm{Bi}$ and Po nuclei is more favorable in the $x n$ evaporation channel than in the $p x n$ channel, which was extensively used below $Z=82$ (see Ref. [53] for a review on the production of proton-emitting nuclei).

The optimal reactions for production of the lightest $\mathrm{Bi}$ and Po isotopes are also discussed, which could be useful for planning future experiments in this region of strongly fissile nuclei.

\section{ACKNOWLEDGMENTS}

We thank the UNILAC staff for providing the stable and high-intensity ${ }^{46} \mathrm{Ti},{ }^{50,52} \mathrm{Cr}$, and ${ }^{94,95,98} \mathrm{Mo}$ beams. This work was supported by the Access to Large Scale Facility program under the Training and Mobility of Researchers program of the European Union within Contract HPRI-CT-1999-00001, by the EXOTAG Contract HPRI-1999-CT-50017, by the FWO-Vlaanderen and the Interuniversity Attraction Poles Programme, Belgian State, Federal Office for Scientific, Technical and Cultural Affairs (IAP Grant P5/07), by UK EPSRC, and by the NSERC of Canada. A.N.A. is especially indebted to the late Dr. D. D. Bogdanov for many fruitful discussions.
[1] S. Cohen, F. Plasil, and W. J. Swiatecki, Ann. Phys. (NY) 82, 557 (1974).

[2] A. J. Sierk, Phys. Rev. C 33, 2039 (1986).

[3] M. Beckerman and M. Blann, Phys. Rev. C 17, 1615 (1978).

[4] A. Gavron, Phys. Rev. C 21, 230 (1980).

[5] W. Reisdorf et al., Nucl. Phys. A438, 212 (1985).

[6] D. Ward et al., Nucl. Phys. A403, 189 (1983).

[7] C.-C. Sahm et al., Nucl. Phys. A441, 316 (1985); J. G. Keller et al., ibid. A452, 173 (1986).

[8] W. Reisdorf, Z. Phys. A 300, 227 (1981); W. Reisdorf and M. Schädel, ibid. 343, 47 (1992); W. Reisdorf, HIVAP manual (GSI, Darmstadt, unpublished).

[9] A. B. Quint et al., Z. Phys. A 346, 119 (1993); A. B. Quint, Ph.D. thesis, TH Darmstadt, GSI-89-23, 1989.

[10] A. N. Andreyev et al., Yad. Fiz. 58, 791 (1995); A. N. Andreyev et al., Phys. At. Nucl. 58, 730 (1995); A. N. Andreyev et al., Nucl. Phys. A583, 169 (1995).

[11] A. N. Andreyev et al., Yad. Fiz. 56, 9 (1993); A. N. Andreyev et al., Phys. At. Nucl. 56, 1302 (1993).

[12] A. V. Yeremin et al., Nucl. Instrum. Methods A 350, 608 (1994); A. N. Andreyev et al., Nucl. Phys. A620, 229 (1997).

[13] R. N. Sagaidak et al., Phys. Rev. C 68, 014603 (2003).

[14] D. J. Hinde et al., Nucl. Phys. A452, 550 (1986); J. O. Newton et al., ibid. A483, 126 (1988); D. J. Hinde, D. Hilscher, and H. Rossner, ibid. A538, 243 (1992).

[15] A. Grewe et al., Nucl. Phys. A614, 400 (1997).

[16] Y. LeBeyec et al., Phys. Rev. C 14, 1038 (1976).

[17] R. L. Hahn, K. S. Toth, Y. LeBeyec, B. Lagarde, and M. W. Guidry, Phys. Rev. C 36, 2132 (1987).

[18] G. Münzenberg et al., Nucl. Instrum. Methods 161, 65 (1979); S. Hofmann and G. Münzenberg, Rev. Mod. Phys. 72, 733 (2000).

[19] In fact, a larger series of the complete-fusion reactions of ${ }^{100} \mathrm{Mo}$ ions with the ${ }^{92-100}$ Mo targets, leading to ${ }^{192-200} \mathrm{Po}^{*} \mathrm{CN}$ nuclei, were studied at SHIP [9].

[20] A. N. Andreyev et al., Nature 405, 430 (2000).

[21] R. Julin, K. Helariutta, and M. Muikku, J. Phys. G 27, 109(R) (2001).

[22] G. D. Dracoulis, G. J. Lane, A. P. Byrne, T. Kibédi, A. M. Baxter, A. O. Macchiavélli, P. Fallon, and R. M. Clark, Phys. Rev. C 69, 054318 (2004).

[23] A. N. Andreyev et al., Eur. Phys. J. A 18, 55 (2003).

[24] A. N. Andreyev et al., Eur. Phys. J. A 6, 381 (1999).
[25] K. Van de Vel et al., Phys. Rev. C 68, 054311 (2003).

[26] K. Van de Vel et al., Eur. Phys. J. A 24, 57 (2005).

[27] A. N. Andreyev et al. (to be published).

[28] K.-H. Schmidt et al., Z. Phys. A 284, 431 (1978); S. Hofmann et al., ibid. 291, 53 (1979).

[29] S. Saro et al., Nucl. Instrum. Methods A 381, 520 (1996).

[30] A. N. Andreyev et al., Eur. Phys. J. A 10, 129 (2001).

[31] A. N. Andreyev et al., Phys. Rev. C 69, 054308 (2004).

[32] A. N. Andreyev et al., Phys. Rev. C 66, 014313 (2002).

[33] P. Van Duppen et al., Nucl. Phys. A529, 268 (1991).

[34] A. N. Andreyev et al., J. Phys. G 25, 835 (1999).

[35] H. Kettunen et al., Eur. Phys. J. A 16, 457 (2003).

[36] K. Helariutta et al., Phys. Rev. C 54, 2799(R) (1996).

[37] A. N. Andreyev et al. (to be published).

[38] D. R. Wiseman et al. (to be published).

[39] M. Hillman and Y. Eyal, JULIAN, Brookhaven National Laboratory Report No. 22846, 1976 (unpublished); O. B. Tarasov and D. Bazin, Nucl. Instrum. Methods B 204, 174 (2004).

[40] M. Blann, Report C00-3494-29, 1976 (unpublished); F. Plasil, Report ORNL-TM-6054, 1977; Phys. Rev. C 17, 823 (1978).

[41] W. Reisdorf et al., Phys. Rev. Lett. 49, 1811 (1982).

[42] L. C. Vaz, J. M. Alexander, and G. R. Satchler, Phys. Rep. 69, 373 (1981).

[43] M. Beckerman, Phys. Rep. 129, 145 (1985); A. B. Balantekin and N. Takigawa, Rev. Mod. Phys. 70, 77 (1998); M. Dasgupta et al., Annu. Rev. Nucl. Part. Sci. 48, 401 (1998).

[44] D. L. Hill and J. A. Wheeler, Phys. Rev. 89, 1102 (1953).

[45] R. Bass, Phys. Rev. Lett. 39, 265 (1977); R. Bass, in Proceedings Symposium on Deep-Inelastic and Fusion Reactions with Heavy Ions, Berlin, 1979 (Springer, Berlin, 1980), p. 281.

[46] A. V. Ignatuk et al., Yad. Fiz. 21, 485 (1975) [Sov. J. Nucl. Phys. 21, 255 (1975)] .

[47] G. Audi and A. H. Wapstra, Nucl. Phys. A595, 509 (1995).

[48] P. Nieminen et al., Phys. Rev. C 69, 064326 (2004).

[49] P. T. Greenlees (private communication).

[50] A. Hürstel et al., Eur. Phys. J. A 21, 365 (2004).

[51] C. N. Davids et al., Phys. Rev. Lett. 76, 592 (1996); G. L. Poli et al., Phys. Rev. C 63, 044304 (2001).

[52] A. N. Andreyev et al., Phys. Lett. B312, 49 (1993).

[53] P. J. Woods and C. N. Davids, Annu. Rev. Nucl. Part. Sci. 47, 541 (1997). 